\title{
Capital social e pobreza no Brasil
}

\author{
Social capital and poverty in Brazil
}

\author{
LILIAN LOPEZ RIBEIRO* \\ JAIR ANDRADE DE ARAUJO**
}

RESUMO: O artigo analisa o impacto do capital social na renda e na redução da pobreza no Brasil. Por meio da técnica dos componentes principais e tendo como base de dados a World Values Survey, construiu-se um índice de capital social considerando três categorias de indicadores: participação em organizações/associações; densidade do sócio e confiança nas pessoas. Concluiu-se que além do capital social contribuir positivamente com a renda, seu impacto é maior que os impactos causados, por exemplo, pelo fato do indivíduo estar empregado, ser casado ou ser de cor branca. Além disso, quanto menor a escala de renda, maior o impacto do capital social na geração de renda e consequentemente na redução da pobreza. PALAVRAS-CHAVE: Capital social; coesões sociais; pobreza.

ABSTRACT: The article analyzes the impact of social capital on income and poverty reduction in Brazil. Through the main components technique and based on the World Values Survey, a social capital index was constructed considering three categories of indicators: participation in organizations/associations; density of the partner and trust in people. It was concluded that in addition to social capital contributing positively with income, its impact is greater than the impacts caused, for example, by the fact that the individual is employed, married or white. In addition, the lower the income scale, the greater the impact of social capital on income generation and consequently on poverty reduction.

KEYWORDS: Capital social; social cohesions; poverty.

JEL Classification: I31.

\section{INTRODUÇÃO}

A contribuição das redes sociais, fundamentadas em relações de confiança, reciprocidade e solidariedade, denominadas de capital social, no aumento do bem-

\footnotetext{
* Professora Adjunta de Economia e Finanças e Pesquisadora. Universidade Federal do Ceará - UFC, campi Sobral. E-mail: liadiniz-21@hotmail.com.

** Professor e Pesquisador no Departamento de Economia Agrícola na Universidade Federal do Ceará, Fortaleza/CE, Brasil. E-mail: jaraujoce@gmail. Submetido: 31/Janeiro/2016; Aprovado: 5/Março/2018.
} 
-estar vem sendo estudada desde há exatos cem anos com o trabalho precursor de Hanifan (1916). Contudo, as Teorias Social e Econômica revelam que a importância desses atributos comportamentais nas relações interpessoais é atemporal.

Veja, por exemplo, o caso do Dilema do agricultor de David Hume e do Dilema dos prisioneiros da Teoria dos Jogos. A solução para esses dilemas é resultante de dois fortes atributos que compõem o capital social, conforme acima ressaltado: a confiança e a cooperação. Até mesmo um clássico problema da Teoria dos Jogos, a "Tragédia dos Comuns", baseado puramente em comportamentos individualistas, poderia ter um resultado desejável para os agentes envolvidos a partir de ação cooperativa entre os mesmos.

Nas últimas duas décadas, sobretudo a partir do trabalho de Putnam et al. (1993), vários pesquisadores como Grootaert (1999), Datt e Jolliffe (1999), Solow (2000) e Tonella (2003), passaram a estudar os efeitos que as redes sociais podem exercer na pobreza. Segundo Wallis et al. (2003), o próprio Banco Mundial, que anteriormente promovia uma abordagem predominantemente neoliberal, tem reconhecido o capital social como uma importante ferramenta para a redução da pobreza.

Não obstante, Toledo (2005) ressalta que a vasta maioria dos pesquisadores no mundo dedicados a estudar a pobreza ainda ignoram a contribuição que o capital social pode ter na privação de renda dos indivíduos. No Brasil, embora o tema já venha sendo explorado por estudiosos da área da sociologia, o capital social, ao contrário do caso de vários países no mundo, não se configura até o momento como objeto de pesquisa entre os economistas, sendo esta uma importante motivação desse estudo.

Outras motivações repousam na constatação de evidências empíricas de que o capital social pode ter um impacto tão relevante ou até mais forte na redução da pobreza quanto os impactos gerados pelos capitais físico e humano, e em acreditar que estudos sobre o capital social podem vir a ser importantes instrumentos de direcionamento de políticas públicas que visam ao bem-estar social.

Diante dessas assertivas, o propósito deste artigo é verificar o efeito do capital social na renda e, consequentemente, na pobreza no Brasil. Como metodologia, utilizou-se a técnica dos componentes principais para construir um índice de capital social, denominado de ICS, com base em três categorias de indicadores: participação em organizações/associações; densidade do sócio e confiança nas pessoas, a partir dos dados disponibilizados pela World Values Survey. Após a construção desse índice, estimou-se o modelo Probit ordenado seguido de seus efeitos marginais.

Dentre os resultados obtidos, verifica-se que além do capital social contribuir positivamente com a renda, seu impacto é superior aos impactos causados pelo emprego, casamento ou pelo fato do indivíduo pertencer à raça branca. Após estimar os efeitos marginais do modelo Probit, percebe-se também que quanto menor a escala de renda, maior o impacto do capital social na geração de renda e, como consequência, na redução da pobreza.

Além da introdução, este artigo apresenta mais quatro outras seções. A segunda faz uma revisão da literatura sobre o capital social, enfatizando o conceito e sua 
relevância, além de apresentar as evidências empíricas. Na terceira seção tem-se a descrição da construção do índice de capital social e dos indicadores utilizados para a sua construção, bem como a apresentação do modelo econométrico estimado. Já a quarta, apresenta os resultados produzidos e sua análise. Na última seção têm-se as considerações finais da pesquisa.

\section{CAPITAL SOCIAL: CONCEITO, RELEVÂNCIA, CRÍTICAS E SUA RELAÇÃO COM A POBREZA}

O trabalho seminal sobre capital social é atribuído a Hanifan (1916) que, ao analisar empiricamente o desempenho de uma escola nos Estados Unidos, constatou que este poderia ser expandido se no cotidiano dos agentes envolvidos houvesse reciprocidade e companheirismo. Posteriormente, nas décadas de 1950 e 1960, o tema foi retomado por um grupo de sociólogos e em 1977 pelo economista Gleen Loury. Contudo, o tema obteve maior popularidade nos anos 1990, sobretudo a partir da publicação do trabalho de Putnam et al. (1993) ${ }^{1}$ (WALLIS et al., 2003).

O capital social pode ser definido como resultante de uma coesão social ou redes sociais informais com objetivos comuns, pautados em normas com alguns atributos como cooperação mútua, confiança, solidariedade, reciprocidade e tolerância. Essas interações, por sua vez, tendem a gerar externalidades positivas, aumentando a disponibilidade de informações, reduzindo as incertezas e os custos de transação, minimizando o oportunismo e podendo até facilitar o fornecimento de bens públicos, propiciando assim ganhos econômicos e de bem-estar para os agentes envolvidos (Putnam et al., 1993; Coleman, 1998; Johannes, 2009; Tenzin et al., 2013).

Não obstante, algumas organizações internacionais como a Organização para Cooperação e Desenvolvimento Econômico (OCDE) e o Banco Mundial têm definido o capital social a partir de suas próprias perspectivas. Enquanto para a OCDE o capital social refere-se a um conjunto de redes sociais com normas, valores e entendimentos que facilitam a cooperação entre grupos, para o Banco Mundial tal capital é representado por instituições, relações e normas que moldam a qualidade e a quantidade de interações sociais da sociedade (Tenzin et al., 2013).

Para Grootaert (2001), apud Andriani e Karyampas (2015), em nível macro o capital social pode ser considerado como sendo um bem social, derivado de redes

\footnotetext{
${ }^{1}$ Em linhas gerais, ao analisar a participação cívica e o desempenho institucional entre as regiões do Norte e do Sul da Itália, Robert Putnam, com a colaboração Robert Leonard e Raffaella Nanetti, observou que diferenças de capital social resultaram em diferenças na boa governança e no crescimento da renda entre as regiões. A pesquisa que durou vinte anos (1970-1990) revelou que as regiões que mostraram melhor desempenho institucional e cívico não coincidiram com regiões que receberam mais recursos ou investimentos e sim com aquelas que praticaram laços fortes de confiança mútua, democracia participativa, transparência administrativa, além de solidariedade e tolerância interpessoal.
} 
que compartilham valores e normas que produzem benefícios mensuráveis para os seus membros, induzindo direta ou indiretamente a um maior nível de bem-estar para um país ou região.

Essa modalidade de capital pode ainda - acrescentam Andriani e Karyampas (2015) e Narayan e Pritchett (1997) - promover um maior e melhor fluxo de informação entre devedor e credor no mercado de crédito e entre o agente e o principal no mercado de trabalho, reduzindo assim as falhas de mercado e comportamentos free-rider.

Já para Peñaloza (2013), o capital social facilita a microcooperativa de negócios, podendo assim ajudar a aumentar a renda temporária dos participantes da rede. Além disso, Hassan e Birungi (2011) argumentam que tal coesão social pode também induzir a um controle eficiente da prestação de bens e serviços do governo, ou ainda atuar como provedor de bens e serviços que o mercado ou o governo não pode fornecer diretamente.

Johannes (2009) considera que um agregado familiar possui uma dotação de ativos constituídos por capital físico, humano e social e que ao combinar tais ativos gera renda e consumo para seus membros. Nessa perspectiva, Toledo (2005) considera que o capital social um importante recurso para combater ou pelo menos amenizar a pobreza, sendo as redes sociais um dos principais meios pelo qual a população pobre da América Latina tem se defendido das vulnerabilidades do mercado.

Semelhantemente concepção de Johannes (2009), Putterman (1995) apud Morris (1998) considera o capital social também como um ativo, porém expandido do capital humano e ampliado para considerar suas dimensões sociais, culturais e a importância da aprendizagem informal. Nessa perspectiva, o acúmulo de capital social aumenta o capital humano e facilita o desenvolvimento econômico de um país.

Por outro lado, Morris (1998) acrescenta que o baixo grau de desenvolvimento de alguns países pode estar intimamente relacionado com a falta de um forte atributo do capital social: a confiança mútua entre os agentes. Afinal, o componente confiança aumenta a qualidade dos relacionamentos entre os agentes, tornando as trocas comerciais mais eficientes, reduzindo assim os custos de transações.

Nessa mesma direção, Wallis et al. (2003) expõem que mesmo que duas economias distintas tenham estoques equivalentes de capital físico e humano, a produtividade dessas economias pode divergir devido à diferença nos graus de confiança. Isso porque, em sociedades com altos níveis de confiança interpessoal e institucional, o capital social pode promover o desenvolvimento de instituições eficazes e economias de escala na produção. Além disso, Knack (2002) complementa que a desigualdade de renda também tende a diminuir nas sociedades com maior grau de confiança.

Apesar da reconhecida importância que o capital social exerce no desenvolvimento econômico de um país ou região, algumas críticas são creditadas a tal capital. Toledo (2005), por exemplo, argumenta que o conceito de capital social está relacionado muitas vezes a ambiguidades e problemas de medição existentes, dado seu caráter multidisciplinar e a indisponibilidade de dados. 
Outra crítica atribuída ao capital social é que segundo Tenzin et al. (2013) e Wallis et al. (2003) uma rede ou uma coesão social, ao se conectar fortemente entre seus membros, pode induzir a uma exclusão social dos não membros, privando estes últimos de informações sobre oportunidades de trabalho e estudo, por exemplo. Algo que pode produzir um efeito não desejado sob o nível de pobreza.

$\mathrm{Na}$ concepção de Hassan e Birungi (2011) há de se considerar também que as coesões sociais são constituídas, geralmente, por um agrupamento interpessoal do tipo homogêneo. Ou seja, as associações são, em sua grande maioria, integradas por pessoas que compartilham as mesmas características como mesmo nível de renda, educacional, étnico etc. Esse tipo de agrupamento pode gerar o que se poderia chamar de "armadilha do capital social", pois tais associações tenderiam a reforçar o conservadorismo, obtendo assim pouco sucesso na geração de conhecimento e inovação. Portanto, associações do tipo heterogêneas são mais desejáveis que as homogêneas.

\section{Capital Social e Pobreza}

Nos últimos vinte anos, a relação entre capital social e pobreza tem sido objeto de estudo de diversos pesquisadores ao redor do mundo, sobretudo por parte de sociólogos e economistas. Estes estudos têm fortalecido o debate teórico, metodológico e pragmático sobre tal relação. Assim sendo, esta seção apresenta importantes constatações de alguns estudiosos do tema.

Dentre tais constatações tem-se a de Andriani e Karyampas (2015) que, apesar de reconhecerem o capital social como um recurso capaz de melhorar o acesso ao mercado de trabalho e de aferir de melhores ganhos monetários, contribuindo assim para a redução da pobreza, atestam que não se pode ignorar a teoria esboçada por Woolcock $(1998,2001)$ com respeito aos laços horizontais fortes e fracos existentes nas redes sociais.

De acordo com essa teoria, redes sociais integradas por grupos familiares ou de parentes - denominados de "laços fortes" - tendem a não serem suficientes para seus integrantes escaparem de uma situação de pobreza. No entendimento de Narayana e Woolcock (2000), esse fator se dá pela ocorrência de um cenário denominado como "armadilha da aproximação", ocasionado por uma possível limitação de recursos e de informação disseminada por parte de uma coesão fechada, caracterizada por este tipo de laço. Deste modo, a suavização da pobreza por meio da formação de capital social é factível a partir dos chamados "laços fracos" - coesão entre pessoas heterogêneas - , estes laços devem ser encorajados no sentido de promover uma maior diversificação e disseminação, sobretudo de conhecimento e de informações.

Por sua vez, Helal e Neves (2007) destacam que na literatura existente acerca da utilização do capital social para o combate, ou suavização, da pobreza coexistem duas perspectivas: a macroscópica e a microscópica. A primeira pressupõe que o impacto do capital social na redução da pobreza ocorre a partir de políticas públicas direcionadas ao incentivo à formação de associações capazes de intensificar a 
integração social entre os participantes. Nessa perspectiva, o capital social seria próprio da comunidade e não do indivíduo. Na segunda, o mecanismo de redução da pobreza se daria por meio dos benefícios que as pessoas obtêm individualmente das redes de relacionamentos que possuem.

No estudo da correlação entre capital social e pobreza, além de destacar a influência do capital social na pobreza, há de se considerar também a influência dessa última na formação de primeira. Sobre esse aspecto, Peña e Proenza (2009) expõem que um baixo estoque de capital social por parte de alguns indivíduos pode ser fruto da pobreza ou miséria, devido a uma limitação ou mesmo desconexão que pessoas muito pobres têm com uma rede de relacionamentos capaz de propiciar melhores condições de vida, uma vez que as coesões sociais são uma medida inclusiva entre os seus integrantes e excludente para aqueles que não a integram.

Não obstante, Portales (2014) salienta que um dos aspectos da relação entre capital social e pobreza se baseia nos efeitos gerados do primeiro no segundo, dando aos pobres uma maior oportunidade de se capacitarem com base na confiança e no reconhecimento entre eles, além de se autogerenciarem e reorganizarem seus próprios interesses e objetivos a fim de melhorar suas condições de vida.

Ainda sobre tal relação, um estudo da Unesco (2002) aborda dois relevantes questionamentos, a saber: pessoas pobres têm o mesmo tipo de capital social que as não pobres? E, as pessoas pobres podem entrar numa coesão social dos não pobres? Para ambos os questionamentos, os autores do estudo são categóricos em responder que não. Segundo estes, a estratificação social e educacional entre os indivíduos se configuram como uma barreira à entrada dos mais pobres em coesões formadas predominantemente por não pobres, excluindo os primeiros de uma coalizão com os segundos. Todavia, o estudo recomenda que a formação de capital social entre os pobres seja ampliada.

Por fim, Collier (1998) apud Portales (2014) argumenta que, em razão da escassez de capital econômico e humano da população pobre, o capital social é considerado um capital constante e inesgotável que pode ajudá-la a garantir o mínimo necessário para viver e, portanto, combater a pobreza. Por essa razão, Garcia (2004) sugere que as instituições públicas incentivem o crescimento da associatividade e reciprocidade, sobretudo, das camadas mais pobres da sociedade.

\section{Evidências Empíricas}

Conforme mencionado na seção anterior, diversos pesquisadores têm se dedicado a estudar os efeitos do capital social na pobreza, especialmente nas economias em desenvolvimento. Isto posto, é relevante citar e apresentar os principais resultados de algumas dessas pesquisas, sobretudo as mais recentes. Em ordem cronológica, têm-se os trabalhos de Peñaloza (2013), Tenzin et al. (2013), Kimsun (2012), Ijaiya et al. (2012), Hassan e Birungi (2011), Andriani e Karyampas (2015), Johannes (2009), Sirven (2006), Toledo (2005), Knack (2002), Morris (1998) e Narayan e Pritchett (1997).

Iniciando com a pesquisa de Knack (2002), as estimações a partir de dados 
cross-country sobre capital social e desempenho econômico para vários países do mundo, revelaram que para 47 desses países a desigualdade de renda diminui nas sociedades com maior grau de confiança. A pesquisa revela que para cada aumento de oito ou nove pontos percentuais no nível de confiança interpessoal, há um declínio de um ponto percentual no índice de Gini.

Com base nos dados retirados da International Policy Research Institute (IFPRI) e do Banco Mundial em parceria com Uganda Bureau of Statistics, Hassan e Birungi (2011) analisaram se o capital social, definido em termos de participações em organizações sociais, afeta positivamente a renda familiar e reduz a pobreza em Uganda. Os resultados mostraram que de fato maiores níveis de renda e bem-estar nesse país estão diretamente associados ao capital social. Em alguns casos, o impacto do capital social verificou-se ser até mais forte do que o impacto do capital humano.

Nessa mesma direção, usando informações do Bhutan Living Standards Survey (BLSS) do ano de 2012 e empregando um modelo de equações simultâneas, Tenzin et al. (2013) constataram que assim como em alguns casos em Uganda, no Butão, além do estoque de capital social contribuir positivamente para a redução da pobreza, sua influência sobre esta é também mais elevada que a influência causada pela educação. Contudo, Tenzin et al. (2013) ressaltam que promover a geração de capital social sem investimento em capital humano seria uma atitude pouco eficiente por parte do governo.

De modo similar, a pesquisa de Kimsun (2012) buscou analisar os determinantes da pobreza crônica e transitória no Camboja com base nos dados em painel de 793 famílias para quatro anos no período de 2001 a 2011. Um dos resultados dessa pesquisa indicou que a coesão social, ao lado do indicador educação do chefe da família, contribui fortemente para que as famílias cambojanas escapem da pobreza.

Por sua vez, Johannes (2009), por meio de variáveis domiciliares de 2001 e fazendo uso de três indicadores de capital social ${ }^{2}$, constatou para Camarões que o indicador que mede a participação individual em associações locais e o de solidariedade representam, assim como o capital humano, uma forte contribuição para as famílias saírem da pobreza.

Dados de 600 famílias do ano de 2001 da Rural Observatories Network (RON), fornecidos pelo National Institute of Statistics (NIS), resultaram no artigo de Sirven (2006) sobre capital social, pobreza e vulnerabilidade em Madagascar. Sua pesquisa concluiu que um elevado nível de capital social, devido à participação dos indivíduos em atividades sociais dentro da comunidade, está associado a uma menor probabilidade de ser pobre e de estar vulnerável a pobreza.

Na Tanzânia tem-se o trabalho de Narayan e Pritchett (1997) que, apoiado nas informações da Tanzania Social Capital and Poverty Survey (SCPS), construiu

\footnotetext{
${ }^{2}$ Os indicadores citados são: Densidade de sócio, Índice de tomada de decisão e Apoio de rede ou Índice de solidariedade.
} 
uma medida de capital social. Ao combinar essa medida com dados sobre rendimento das famílias, verificou-se que o impacto das redes sociais é tão forte quanto triplicar o nível de educação da população.

Ijaiya et al. (2012) também realizaram um estudo de caso relacionando pobreza e capital social em um país africano, a Nigéria. A pesquisa que contemplou 600 famílias da cidade de Minna e foi retirada dos dados amostrais do African Development Bank Gender de 2003 apontou, assim como na maioria das pesquisas realizadas o capital social, que este se relaciona positivamente com a redução da pobreza.

Um estudo de igual relevância aos já citados é o que Morris (1998) realizou para a Índia. Tendo como fonte de dados o National Sample Survey (NSS) para vários anos e se baseando em um modelo econométrico de redução da pobreza sugeridos por Datt e Ravallion (1996), esse autor incorporou, além dos capitais físico e humano, o capital social na determinação da pobreza dos estados indianos. A pesquisa revelou que aqueles estados com maior dotação inicial de capital social obtiveram um maior sucesso na redução da pobreza.

Outra pesquisa importante é a de Andriani e Karyampas (2015) realizada em nível regional para a Itália e usando dados dos anos de 2002 e 2003. Os resultados produzidos por esse estudo revelam que regiões com um nível mais elevado de capital social tendem, em média, a obter menores taxas de incidência e de intensidade da pobreza. Além disso, a exclusão social (resultante de uma média aritmética das dimensões econômica, social e humana) mostrou-se negativa e significantemente correlacionada com os indicadores de capital social incluídos no modelo. Uma relevante constatação é que o nível de educação, considerado na pesquisa, não se mostrou significativamente correlacionado com as variáveis da pobreza.

Em outra direção, contrariando a maioria dos estudos empíricos já realizados, a pesquisa de Peñaloza (2013) para o Equador, no período de 2010 a 2012, não encontrou evidências empíricas para sustentar que o capital social é uma variável capaz de contribuir com a redução da pobreza. Na concepção de Peñaloza (2013), esse resultado pode estar relacionado a falhas na definição e quantificação do capital social, fatores que podem impedir de mensurar a verdadeira conexão entre esse tipo de capital e a pobreza.

Convergindo com os resultados de Peñaloza (2013), os estudos sobre pobreza e capital social de Toledo (2005) realizado para a capital da argentina indicam que as redes sociais estão longe de ser um instrumento eficaz ou suficiente para evitar o risco de incidência de pobreza. A pesquisa, que é resultante de um banco de dados dos meses de outubro de 2001 a maio de 2002 e oriunda do Gran Buenos Aires (GBA), relacionou dois indicadores de capital social (laços fortes e fracos) ${ }^{3} \mathrm{com}$ as diferentes definições de pobreza (absoluta, extrema e relativa).

\footnotetext{
${ }^{3}$ Em linhas gerais, segundo Toledo (2005), os laços fortes referem-se aos laços familiares e de amigos mais próximos, enquanto os laços fracos são aqueles formados no ambiente de trabalho e em associações em geral.
} 


\section{ESTRATÉGIA METODOLÓGICA}

As variáveis que resultaram nesse estudo foram extraídas do banco de dados da World Values Survey (WVS) para o ano de 20144. A WVS é uma associação não governamental com sede na Suécia e que desde 1981 aplica pesquisas de cunho sociocultural e econômico em quase cem países. O objetivo dessa associação é gerar um banco de dados da evolução de crenças e valores de pessoas do mundo todo, com objetivos de pesquisas sociais para o direcionamento de políticas públicas.

A seguir, a descrição da construção do Índice de Capital Social e das variáveis utilizadas e a apresentação e especificação do modelo econométrico estimado.

\section{Construção do Índice de Capital Social}

Para analisar a influência que o capital social pode exercer na pobreza do Brasil, construiu-se inicialmente um índice agregado de capital social por meio da técnica dos componentes principais. Esta é uma técnica de análise multivariada que permite obter uma combinação linear de variáveis, capturando o máximo de variância possível fornecida pelo conjunto das variáveis selecionadas e tendo como base a decomposição da matriz de correlação R (covariância) ${ }^{5}$.

Nesses termos, seja $\lambda_{n}$ o autovalor, também chamado de raiz característica da matriz $\mathrm{R}$, da $n$-ésima componente principal observada para uma variável qualquer do conjunto de variáveis $X=\left(X_{1,}, \ldots \ldots ., X_{j}\right)$ da amostra, sendo que $\lambda_{1}>\lambda_{2}>\lambda_{3}, \ldots \ldots, \lambda_{j}$. Para cada autovalor existe um autovetor correspondente denotado por $a_{i j}$. Logo, a n-ésima componente principal para uma observação $j$ da amostra é expressa por $C_{n}^{j}=\sum_{j} a_{i j} X_{j}$. Desse modo, para cada observação $j$ tem-se um Índice de Capital Social dado por

$$
I C S_{j}=\frac{\sum_{n} \lambda_{n} c_{n}^{j}}{\sum_{n=1} \lambda_{n}}
$$

Atendendo à regra de Kaiser (1960), que pressupõe considerar apenas componentes com autovalores acima de um, $\lambda>1$, bem como o pressuposto de que a soma das variâncias de tais componentes deve representar uma porcentagem acima de $80 \%$ (Manly, 2008), foram utilizadas as onze primeiras componentes geradas. Tais componentes explicam $86,6 \%$ da covariação entre as variáveis da amostra.

\footnotetext{
${ }^{4} \mathrm{Na}$ pesquisa realizada para o Brasil em 2014 foram entrevistados 1.278 indivíduos.

${ }^{5}$ É oportuno ressaltar também que cada componente principal é uma combinação linear de todas as variáveis originais, que são independentes entre si e estimadas com o propósito de reter, em ordem de estimação, o máximo de informação, em termos da variação total contida nos dados. Ou seja, a análise de componentes principais é associada à ideia de redução de massa de dados, com menor perda possível da informação (Regazzi, 2000).
} 
$\mathrm{Na}$ construção do Índice de Capital Social, por meio da técnica acima especificada, considerou-se um total de trinta variáveis dicotômicas resultantes de perguntas destinadas ao entrevistado que estão relacionadas a organizações ou associações nas quais o indivíduo questionado participa e à confiança que o mesmo atribui às pessoas. Tais variáveis se enquadram nas seguintes três categorias de indicadores:

1. Participação em Organizações/Associações: Indicam se o entrevistado é integrante, porém não participante ativamente. Ou seja, membro não ativo de organizações/associações de igreja ou grupo de religião; esportiva ou recreativa; artística, musical ou educacional; sindicato; partido político; de meio ambiente; profissional; humanitária ou de caridade; de consumidores; grupo de autoajuda, grupo de ajuda mútua e outra organização.

2. Densidade do Sócio: Apontam se o entrevistado é integrante de organizações/associações e participa ativamente de tais entidades. Ou seja, membro ativo de organizações/associações de igreja ou grupo de religião; esportiva ou recreativa; artística, musical ou educacional; sindicato; partido político; de meio ambiente; profissional; humanitária ou de caridade; de consumidores; grupo de autoajuda, grupo de ajuda mútua e outra organização.

3. Confiança nas Pessoas: Indicam se o entrevistado confia nas pessoas em geral: na sua família; seus vizinhos; pessoas que conhece pessoalmente; pessoas que está vendo pela primeira vez; pessoas de outras religiões e pessoas de outros países. Além desses, também o subindicador autoconfiável, para identificar se o entrevistado se vê como alguém que é geralmente confiável e o indicador de oportunismo, em que o entrevistado é questionado se diante de uma chance a maioria das pessoas tentariam levar vantagem sobre ele ou tentariam ser justas.

Uma justificativa para a escolha de tais indicadores como proxies para o capital social pode ser vislumbrada em Hassan e Birungi (2011) ao ressaltar que a participação em organizações locais, por exemplo, tem sido um indicador amplamente utilizado nos estudos sobre capital social, por ser sugerida por Putnam et al. (1993) e por ser de fácil introdução, algo que facilita a comparação com os demais estudos. Além disso, tais indicadores dependem de valores comuns atribuíveis ao capital social como respeito mútuo, confiança, reciprocidade e cooperação, representando, portanto, adequadas proxies para a construção do índice de capital social.

Além do ICS, as demais variáveis usadas na estimação do modelo econométrico especificado a seguir são: a escala de renda e idade do entrevistado, acrescido de dezesseis dummies construídas a partir das seguintes variáveis: ocupação; nível educacional mais alto; estado civil; sexo; grupo étnico e regiões brasileiras.

\section{Especificação do Modelo Econométrico}

Para a análise empírica do impacto do capital social na renda e, consequentemente, na pobreza do Brasil é utilizado o modelo Probit ordenado seguido de seus 
efeitos marginais. Esse modelo pode ser visto como um modelo de regressão linear para uma variável resposta contínua não observada (latente) na forma:

$$
y_{i}^{*}=X_{i} \beta_{i}+\varepsilon_{i}
$$

sendo " $i$ " o número de cada observação, $y_{i}$ é uma variável discreta a ser estimada a partir de um vetor de variáveis explicativas representadas por $X_{i}$, em que o parâmetro $\beta_{i}$ é o coeficiente desse vetor de variáveis. Assume-se também que o erro aleatório, $\varepsilon_{i}$, segue uma distribuição Normal com média zero e variância um, ou seja, $X_{i} \sim \mathrm{N}(0,1)$.

\section{RESULTADOS GERADOS}

A Tabela 1 apresenta os resultados estimados da regressão Probit para a determinação dos níveis de renda dos indivíduos entrevistados pela WVS (considerando as categorias de menor renda relativa às de maior renda) tendo como variáveis explicativas o índice de capital social, ICS, bem como demais variáveis controle representadas pelas características pessoais dos indivíduos. É pertinente salientar que como o objetivo da pesquisa é verificar como o capital social influi na renda e, consequentemente, na pobreza, a análise será feita com foco na variável explicativa ICS.

Primeiramente, ao analisar o p-valor, é possível observar pela tabela abaixo que o capital social, representado pelo ICS, apresenta-se estatisticamente significante na determinação da renda no nível de 1,2\%. Ao comparar esse resultado estatístico com aqueles encontrados pelas demais variáveis consideradas na estimação, percebe-se que o nível de significância do capital social em explicar o nível de renda é maior, por exemplo, que o fato do indivíduo possuir curso superior, ser casado ou residir na região Sudeste ou Sul do país, comparado com os que vivem no Centro-oeste.

Pela Tabela 1, é possível obter também o impacto do capital social e das demais variáveis explicativas no nível de renda por meio da razão de probabilidades, expressas pela $\exp \left(\beta_{K}\right)$ das referidas variáveis.

Mediante essa análise constata-se que o capital social pode contribuir para o aumento no nível de renda por um fator de $1,7 \mathrm{vez}$. Ao considerar as demais variáveis que se mostraram estatisticamente significantes, esse impacto positivo gerado pelo capital social é ligeiramente superior àqueles obtidos pelo fato de estar empregado $(1,4)$ quando confrontados a outros tipos de ocupação: estudantes, donas de casa e aposentados, de ser casado $(1,1)$ ou de ser de raça branca $(1,2)$, comparado a outros tipos de raça - indígena, parda e amarela. Para indivíduos com curso superior o impacto positivo na renda tende a ser dado por um fator de 4,2 vezes. 
Tabela 1: Regressão Probit para a Variável Renda no Brasil

\begin{tabular}{lcccc}
\hline \multicolumn{1}{c}{ Variáveis } & Coef. & Desv.-Pad. & Z & Valor-p \\
\hline ICS $_{i}$ & 0.545 & 0.217 & 2.51 & 0.012 \\
Idade $_{i}$ & -0.036 & 0.010 & -3.61 & 0.000 \\
Idade $_{i}$ & 0.0003 & 0.0001 & 3.28 & 0.001 \\
Dempr $_{i}$ & 0.331 & 0.073 & 4.48 & 0.000 \\
Ddesempr $_{i}$ & 0.124 & 0.100 & 1.23 & 0.217 \\
Dsup $_{i}$ & 1.445 & 0.627 & 2.31 & 0.021 \\
Dfundmédio $_{i}$ & 1.091 & 0.619 & 1.76 & 0.078 \\
Dmasc $_{i}$ & -0.056 & 0.064 & -0.88 & 0.381 \\
Dcas $_{i}$ & 0.132 & 0.062 & 2.12 & 0.034 \\
Dbran $_{i}$ & 0.205 & 0.067 & 3.04 & 0.002 \\
Dnegr $_{i}$ & -0.041 & 0.098 & -0.42 & 0.675 \\
DNO $_{i}$ & -0.060 & 0.145 & -0.42 & 0.676 \\
DNE $_{i}$ & -0.605 & 0.119 & -5.05 & 0.000 \\
DSE $_{i}$ & -0.204 & 0.114 & -1.78 & 0.075 \\
DSUL $_{i}$ & -0.217 & 0.131 & -1.65 & 0.098 \\
\hline
\end{tabular}

Fonte: Estimativas dos autores.

Dando sequência à análise, os resultados contidos na Tabela 2, representados pela estimação dos efeitos marginais, demonstram a magnitude do impacto gerado por cada variável explicativa na renda desagregada em dez escalas que vai de um (menor nível de renda) a dez (maior nível). É possível observar em quanto varia a probabilidade de ter maior ou menor nível de renda, quando ocorre uma variação unitária em uma dessas variáveis, mantidas as demais constantes.

Em relação ao efeito da idade das pessoas sobre o nível de renda, os resultados reforçam o fato de que sua trajetória não é constante ao longo da vida. Inicialmente, com o aumento da idade, a probabilidade de ter renda baixa aumenta para as categorias de indivíduos com escala de renda mais baixa e, posteriormente, a partir de uma idade mais avançada, essa probabilidade começa a cair. Na mesma direção, para indivíduos com escala de renda mais elevada, aqueles a partir da quinta escala, mais anos de vida reduzem a chance de ter maior nível de renda, mas depois de certa idade há um aumento nessa probabilidade. O comportamento dessa variável é captado pelos regressores $\operatorname{Idade}_{i}$ e Idade $2_{i}$.

Conforme já esperado, as variáveis explicativas relacionadas à ocupação (Dem$\left.p r_{i}\right)$, ao estado civil $\left(\right.$ Dcas $\left._{i}\right)$ e à raça $\left(\right.$ Dbran $\left._{i}\right)$, contribuem positivamente para a probabilidade de aumento do nível de renda, independentemente da escala de rendimento. Por outro lado, não se evidenciou variações na renda dada às variações dos

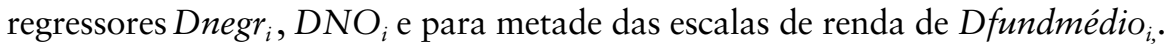

O mesmo não ocorre com a variável $I C S_{i}$. Ao centrar a análise na variação desse índice é possível notar que, mantendo constantes as demais variáveis explicativas, um aumento no capital social diminui as chances de indivíduos com menores níveis 
de renda, representados pelas categorias com escalas de renda de um a quatro, auferir baixos rendimentos. Ou seja, a coesão social na forma de adesão e participação em associações ou organizações pode, por exemplo, diminuir cerca de $10 \%$ a probabilidade do grupo de indivíduos com a menor escala de renda a continuar a ter baixos níveis de rendimento. Para as três categorias seguintes com menor escala de renda, essa diminuição é sentida em torno de $5,4 \% ; 3,4 \%$ e $2,2 \%$, respectivamente.

Já os efeitos marginais para as demais categorias, a partir da quinta escala de renda, mostram que os acréscimos de capital social fazem com que a probabilidade de obter maior renda cresça. No caso da categoria com escala de renda de nível seis, esse aumento na renda é em 6,4\%. Entretanto, os aumentos marginais na renda provocados por essa modalidade de capital se mostram decrescentes de uma escala de renda para a outra, chegando a ser insignificante para a categoria com maior nível de renda, décima escala, conforme exposto na tabela abaixo.

Dado que a categoria com menor nível de rendimento, escala de nível um, responde mais sensivelmente ao estímulo impulsionado pelo incremento de capital social que a categoria da segunda escala e assim sucessivamente até a quarta escala, é plausível supor que quanto menor a renda, maior o impacto do capital social na geração de renda e consequentemente na redução da pobreza. Contudo, é importante mencionar que essa contribuição do capital social parece ser menor que aquela oriunda do capital humano, representado na tabela a seguir pela dummy que indica se o entrevistado possui curso superior completo, Dsup $p_{i}$, comparado aos indivíduos não alfabetizados (categoria base). Observe a tabela a seguir.

Tabela 2: Efeitos Marginais para a Probabilidade de Renda no Brasil

\begin{tabular}{lcccccccc}
\hline \multicolumn{1}{c}{ Variáveis } & $\begin{array}{c}\mathrm{d} y / \mathrm{d} x \\
(1)\end{array}$ & $\begin{array}{c}\text { Valor-p } \\
(1)\end{array}$ & $\begin{array}{c}\mathrm{d} / \mathrm{d} x \\
(\mathbf{2})\end{array}$ & $\begin{array}{c}\text { Valor-p } \\
(\mathbf{2})\end{array}$ & $\begin{array}{c}\mathrm{d} y / \mathrm{d} x \\
(3)\end{array}$ & $\begin{array}{c}\text { Valor-p } \\
(3)\end{array}$ & $\begin{array}{c}\mathrm{dy} / \mathrm{dx} \\
(4)\end{array}$ & $\begin{array}{c}\text { Valor-p } \\
(4)\end{array}$ \\
\hline ICSi & -0.104 & 0.013 & -0.054 & 0.014 & -0.034 & 0.015 & -0.022 & 0.018 \\
Idadei & 0.007 & 0.000 & 0.003 & 0.001 & 0.002 & 0.001 & 0.001 & 0.001 \\
Idade2i & -0.00007 & 0.001 & -0.00003 & 0.002 & -0.00002 & 0.002 & -0.00001 & 0.003 \\
Dempri & -0.063 & 0.000 & -0.032 & 0.000 & -0.020 & 0.000 & -0.013 & 0.000 \\
Ddesempri & -0.022 & 0.191 & -0.012 & 0.217 & -0.008 & 0.235 & -0.005 & 0.272 \\
Dsupi & -0.139 & 0.000 & -0.103 & 0.000 & -0.089 & 0.000 & -0.101 & 0.008 \\
Dfundmédioi & -0.314 & 0.163 & -0.076 & 0.000 & -0.023 & 0.174 & 0.016 & 0.667 \\
Dmasci & 0.010 & 0.385 & 0.005 & 0.382 & 0.003 & 0.379 & 0.002 & 0.374 \\
Dcasi & -0.025 & 0.038 & -0.013 & 0.037 & -0.008 & 0.035 & -0.005 & 0.034 \\
Dbrani & -0.039 & 0.002 & -0.020 & 0.003 & -0.013 & 0.004 & -0.008 & 0.005 \\
Dnegri & 0.008 & 0.681 & 0.004 & 0.675 & 0.002 & 0.671 & 0.001 & 0.660 \\
DNOi & 0.012 & 0.685 & 0.006 & 0.676 & 0.003 & 0.668 & 0.002 & 0.649 \\
DNEi & 0.135 & 0.000 & 0.057 & 0.000 & 0.031 & 0.000 & 0.013 & 0.000 \\
DSEi & 0.040 & 0.083 & 0.020 & 0.076 & 0.012 & 0.072 & 0.007 & 0.064 \\
DSULi & 0.045 & 0.130 & 0.021 & 0.095 & 0.012 & 0.070 & 0.006 & 0.020 \\
\hline & & & & & & & continua na página seguinte
\end{tabular}




\begin{tabular}{|c|c|c|c|c|c|c|c|c|}
\hline Variáveis & $d y / d x$ & $\begin{array}{c}\text { Valor-p } \\
\text { (5) }\end{array}$ & $d y / d x \quad(6)$ & $\begin{array}{c}\text { Valor-p } \\
(6)\end{array}$ & $\begin{array}{c}d y / d x \\
(7)\end{array}$ & $\begin{array}{c}\text { Valor-p } \\
\text { (7) }\end{array}$ & $\begin{array}{c}d y / d x \\
\text { (8) }\end{array}$ & $\begin{array}{c}\text { Valor-p } \\
\text { (8) }\end{array}$ \\
\hline ICSi & 0.030 & 0.020 & 0.064 & 0.014 & 0.064 & 0.014 & & \\
\hline Idadei & -0.002 & 0.002 & -0.004 & 0.001 & -0.004 & 0.001 & & \\
\hline Idade $2 \mathrm{i}$ & 0.00002 & 0.004 & 0.00004 & 0.001 & 0.00004 & 0.001 & & \\
\hline Dempri & 0.018 & 0.000 & 0.038 & 0.000 & 0.038 & 0.000 & & \\
\hline Ddesempri & 0.005 & 0.114 & 0.014 & 0.207 & 0.014 & 0.229 & & \\
\hline Dsupi & -0.096 & 0.251 & 0.055 & 0.164 & 0.146 & 0.000 & & \\
\hline Dfundmédioi & 0.132 & 0.134 & 0.117 & 0.016 & 0.087 & 0.002 & & \\
\hline Dmasci & -0.003 & 0.398 & -0.006 & 0.383 & -0.006 & 0.380 & & \\
\hline Dcasi & 0.007 & 0.053 & 0.015 & 0.037 & -0.006 & 0.035 & & \\
\hline Dbrani & 0.011 & 0.005 & 0.024 & 0.003 & 0.024 & 0.003 & & \\
\hline Dnegri & -0.002 & 0.694 & -0.004 & 0.677 & -0.004 & 0.672 & & \\
\hline DNOi & -0.003 & 0.703 & -0.007 & 0.679 & -0.007 & 0.671 & & \\
\hline DNEi & -0.049 & 0.000 & -0.071 & 0.000 & -0.064 & 0.000 & & \\
\hline DSEi & -0.012 & 0.105 & -0.024 & 0.077 & -0.023 & 0.073 & & \\
\hline DSULi & -0.0161 & 0.185 & -0.026 & 0.104 & -0.024 & 0.081 & & \\
\hline Variáveis & $\begin{array}{c}d y / d x \\
\text { (9) }\end{array}$ & $\begin{array}{c}\text { Valor-p } \\
\text { (9) }\end{array}$ & $\begin{array}{c}d y / d x \\
(10)\end{array}$ & $\begin{array}{c}\text { Valor-p } \\
\text { (10) }\end{array}$ & & & & \\
\hline ICSi & 0.032 & 0.017 & 0.019 & 0.023 & & & & \\
\hline Idadei & -0.002 & 0.001 & -0.001 & 0.003 & & & & \\
\hline Idade $2 \mathrm{i}$ & 0.00002 & 0.002 & 0.00001 & 0.005 & & & & \\
\hline Dempri & 0.019 & 0.000 & 0.012 & 0.001 & & & & \\
\hline Ddesempri & 0.007 & 0.247 & 0.004 & 0.267 & & & & \\
\hline Dsupi & 0.129 & 0.017 & 0.134 & 0.156 & & & & \\
\hline Dfundmédioi & 0.036 & 0.001 & 0.019 & 0.002 & & & & \\
\hline Dmasci & -0.003 & 0.380 & -0.002 & 0.380 & & & & \\
\hline Dcasi & 0.007 & 0.038 & 0.004 & 0.044 & & & & \\
\hline Dbrani & 0.012 & 0.005 & 0.007 & 0.009 & & & & \\
\hline Dnegri & -0.002 & 0.669 & -0.001 & 0.667 & & & & \\
\hline DNOi & -0.003 & 0.667 & -0.002 & 0.662 & & & & \\
\hline DNEi & -0.030 & 0.000 & -0.017 & 0.000 & & & & \\
\hline DSEi & -0.011 & 0.076 & -0.007 & 0.083 & & & & \\
\hline DSULi & -0.011 & 0.073 & -0.006 & 0.070 & & & & \\
\hline
\end{tabular}

Fonte: Estimativas dos autores. 
As evidências acima apresentadas convergem para os resultados observados por Sirven (2006) em Madagascar, Ijaiya et al. (2012) para a Nigéria e Morris (1998) para a Índia. Já em Hassan e Birungi (2011), Tenzin et al. (2013); Kimsun (2012); Johannes (2009), Narayan e Pritchett (1997) e Andriani e Karyampas (2010), além de ser constatado que o capital social induz a uma redução na pobreza, seu impacto é maior ou pelo menos tão forte quanto a contribuição do capital humano para a Uganda, o Butão, Camboja, Camarões, Tanzânia e Itália, respectivamente.

Embora o capital humano continue a representar um instrumento primordial de combate à pobreza no Brasil, os resultados dessa pesquisa revelam que o capital social pode se configurar, ainda que de modo coadjuvante, como um importante ativo redutor de pobreza ou suavizador das más condições de vida que podem, eventualmente, ocorrer em momentos de recessão econômica.

Diante das assertivas apresentadas neste artigo, sugere-se que as coesões sociais informais, sejam elas culturais, ambientais, profissionais, humanitárias ou afins, baseadas na confiança, cooperação, reciprocidade e solidariedade devem ser estimuladas, pois tais coesões que se convencionou chamar de capital social é certamente, além de uma medida redutora de pobreza, o cerne para um desenvolvimento endógeno e sustentável tão perseguido por países do mundo todo.

\section{CONSIDERAÇÕES FINAIS}

Esse artigo analisou a influência do capital social na renda e, consequentemente, na pobreza no Brasil. Para atender a esse objetivo, estimou-se o modelo Probit e seus efeitos marginais. Mas, previamente, produziu-se por meio da técnica dos componentes principais um índice de capital social, ICS, em que foram ponderadas três categorias de indicadores: participação em organizações/associações; densidade do sócio e confiança nas pessoas, tendo como base os dados disponibilizados pela World Values Survey.

Os resultados revelaram que além do capital social contribuir positivamente com a renda, seu impacto é superior, por exemplo, aos impactos causados pelo emprego, casamento ou pelo fato do indivíduo ser de raça branca. Ou seja, enquanto a razão de probabilidade sugere que o capital social pode aumentar o nível de renda em 1,7 vez, esse aumento no nível de renda dos indivíduos que estão empregados é de apenas 1,4 vez (quando confrontados a outros tipos de ocupação: estudantes, donas de casa e aposentados), de 1,1 vez para aqueles que são casados e de 1,2 vez para indivíduos de raça branca (comparados as raças indígena, parda e amarela).

Por meio dos efeitos marginais, verificou-se que quanto menor a escala de renda, maior o impacto do capital social na geração de renda e consequentemente na redução da pobreza. Um aumento no capital social reduz em $10 \%$ a probabilidade da categoria com menor escala de renda a continuar a obter baixos rendimentos. Para as categorias com escala de renda seguintes, dois, três e quatro, as chances de queda são de $5,4 \% ; 3,4 \%$ e $2,0 \%$, respectivamente. Contudo, a contribuição do 
capital social parece ser menor que aquela oriunda do capital humano, representado pela dummy que indica se o indivíduo possui curso superior completo, comparado aos não alfabetizados.

Enfim, os resultados evidenciam que o capital social representa, ainda que de modo coadjuvante ao capital humano, um importante instrumento para reduzir a pobreza ou para suavizar as más condições de vida que podem, eventualmente, ocorrer em momentos de recessão econômica.

Diante disso, sugere-se que as coesões sociais culturais, ambientais, profissionais, humanitárias e afins, pautadas na confiança, cooperação, reciprocidade e solidariedade devem ser estimuladas, pois tais coesões que se convencionou chamar de capital social são possivelmente, além de uma medida redutora de pobreza, o cerne para um desenvolvimento endógeno e sustentável tão perseguido por países do mundo todo.

\section{REFERÊNCIAS BIBLIOGRÁFICAS}

ANDRIANI L. - KARYAMPAS D., (2015) “Social Capital, Poverty and Social Exclusion in Italy". Revista Debates. Vol $9 \mathrm{n}^{\circ} 2$.

DATT, G.; JOLLIFFE, D. (1999) “Determinants of poverty in Egypt: 1997”. Food Consumption and Nutrition Division Discussion Paper, $\mathrm{n}^{\circ} 75$. Washington, DC: International Food Policy Research Institute - IFPRI.

GARCIA, M. (2004) “Aportes del Capital Social a la Superación de la Pobreza Una Alternativa para México” Journal Razón y palavra. Vol. 37.

GROOTAERT, C., NARAYAN, D., JONES, V. N.; WOOLCOCK, M. (2003) Integrated questionnaire for the measurement of social capital. The World Bank Social Capital Thematic Group. The World Bank.

GROOTAERT, C. (1999) Social Capital, Household Welfare and Poverty in Indonesia. vol. 2148. World Bank Publications. Washington, DC.

GUJARATI. D. (2004) Basic econometrics. 4.ed. Boston: McGraw-Hill.

HASSAN, R.; BIRUNGI, P. (2011) "Social Capital and Poverty in Uganda”. Development Southern Africa, vol. 28, no. 1, pp. 19-37.

HANIFAN, L. J. (1916) “The Rural School Community Center.” Annals of the American Academy of Political and Social Science, $\mathrm{n}^{\circ}$ 67, pg. 130-138.

HELAL, D. H.; NEVES, J. A. B. (2007) "Superando a Pobreza: o Papel do Capital Social na Região Metropolitana de Belo Horizonte." Cadernos EBAPE.BR, Vol. 5, nº 2.

IJAIYA, M. A.; SAKARIYAU, O. B.; DAUDA, C. K.; PAIKO, I. I.; ZUBAIRU, U. M. (2012) "Social Capital and Poverty Reduction in Nigeria: A Case Study of Minna Metropolis”. International Journal of Business and Social Science. Vol. 3, n. ${ }^{\circ} 12$.

JOHANNES, T. A. (2009) "Does Social Capital Determine Poverty? Evidence from Cameroon Household Survey". Global Network for Economics of Learning, Innovation, and Competence Building Systems. Conference: UNU-MERIT.

KAISER, H. F. (1960) “The Application of Electronic Computers to Factor Analysis". Educational and Psychological Measurement, v. 20, p. 141 - 151.

KIMSUN, T. (2012) "Analysing Chronic Poverty in Rural Cambodia: Evidence from Panel Data". Working Paper Series No. 66.

KNACK, S. (2002) “Social capital, Growth and Poverty: a Survey of Cross-Country Evidence.” Cap. 11. MPRA - Munich Personal RePEc Archive. World Bank. 
MANLY, B. J. F. (2008) Métodos Estatísticos Multivariados: uma Introdução. Tradução de Sara Ianda Carmona. 3. ed. Porto Alegre: Bookman.

MORRIS, M. (1998) “Social Capital and Poverty in India”. IDS Working Paper 61. Brighton: Institute for Development Studies.

NARAYAN, D.; PRITCHETT, L. (1999) "Cents and Sociability: Household Income and Social Capital in Rural Tanzania". Economic Development and Cultural Change .Vol. 47, $\mathrm{n}^{\circ} .4$.

PUTNAM, R., LEONARDI, R. AND NANELTI, R. (1993) Making Democracy Work: Civic Traditions in Modern Italy. Princeton, Princeton University Press.

PEÑALOZA, H. A. B. (2013) “Capital Social y Pobreza en Ecuador, 2010-2012”. Universidad Autónoma de Manizales, Vol. 20, $\mathrm{n}^{\circ}$ 35, 93-115.

PORTALES, L. (2014) "Capital social y pobreza multidimensional, el caso de hogares pobres en Monterrey, México”. Convergência. Vol. 21, n $66 .$.

PEÑA, A.; PROENZA, D. (2009) "Capital Social y Pobreza Urbana: el lugar de las instituciones oficiales en la superación de la precariedad en territorios periféricos de Ciudade La Habana, Cuba. “ Revista de Estudos e Pesquisas sobre as Américas, Vol. 2.

REGAZZI, A.J. (2000) “Análise Multivariada, Notas de Aula INF 766”. Departamento de Informática da Universidade Federal de Viçosa, v.2,

SIRVEN, N. (2006) “Social Capital, Poverty and Vulnerability in Madagascar.” Research Associate, Capability and Sustainability Centre, VHI. University of Cambridge, England.

SOLOW, R. M. (2000) "Notes on Social Capital and Economic Performance”, in P. Dasgupta and I. Serageldin (eds) Social Capital: A Multifaceted Perspective. World Bank, Washington, DC.

TENZIN, G.; NATSUDA, K. (2013) "Impact of Social Capital on Poverty: A Case of Rural Households in Eastern Bhutan." RCAPS Working Paper Series, University, Japan.

TOLEDO, F. (2005) Pobreza y Capital Social: Dilucidando los Procesos de una Relación Compleja. Un Análisis para el Caso del GBA. Dissertação (Mestrado em Economia) Universidad Nacional de La Plata. Buenos Ares.

TONELLA, C. (2003) “Capital Social y Reducción de la Pobreza en América Latina y el Caribe: en Busca de un Nuevo Paradigma.” Revista de Sociologia e Política, n² 21, p.187-190.

UNESCO. (2002) Social Capital and Poverty Reduction: Wich role for the civil society organizations and state? United Nations Educational, Scientific and Cultural Organization. Paris.

WALLIS, J.; KILLERBY, P.; DOLLERY, B. (2003) “Social Economics and Social Capital.” Working Paper Series in Economics, ${ }^{\circ}$ 2003-4. School of Economics, University of New England.

WOOLCOCK, M. (1998) "Social capital and economic development: toward a theoretical synthesis and policy framework", Theory and Society, Vol. 27, $\mathrm{n}^{\circ} 2$.

WOOLCOCK, M. (2001) "The place of social capital in understanding social and economic outcomes", Canadian Journal of Policy Research, Vol. 3. 